will be published in Astronomy Reports, 2018, vol.62

\title{
To the problem of spectral mimicry of supergiants
}

\author{
V.G. Klochkova and E.L. Chentsov \\ Special Astrophysical Observatory RAS, Nizhnij Arkhyz, 369167 Russia
}

August 1, 2018

\begin{abstract}
The phenomenon of spectral mimicry refers to the fact that hypergiants and post-AGB supergiants - stars of different masses in fundamentally different stages of their evolution have similar optical spectra, and also share certain other characteristics (unstable and extended atmospheres, expanding gas-dust envelopes, high IR excesses). As a consequence, it is not always possible to distinguish postAGB stars from hypergiants based on individual spectral observations in the optical range. Examples of spectral mimicry are presentes using uniform, high-quality spectral material obtained on the 6-m telescope of the Special Astrophysical Observatory in the course of long-term monitoring of high-luminosity stars. It is shown that unambiguously resolving the mimicry problem for individual stars requires the determination of a whole set of parameters: luminosity, wind parameters, spectral energy distribution, spectral features, velocity field in the atmosphere and circumstellar medium, behavior of the parameters with time, and the abundances of chemical elements in the atmosphere.

Keywords: stars, evolution, supergiants, hypergiants, AGB stars, circumstellar envelopes, optical spectroscopy.
\end{abstract}

\section{Introduction}

Numerous massive Population I supergiants have very well defined, similar spectra that can be used to identify them fairly certainly. The spectra of the small number of known hypergiants are peculiar, with this peculiarity varying widely from object to object. The spectra of low-mass post-AGB supergiants differ appreciably from the spectra of massive Population I supergiants, but their spectra are often difficult to distinguish from those of hypergiants. Thus, given the uncertainties in the estimated distances for Galactic objects, an object with a powerful wind but with a lower mass and luminosity can be mistaken for a hypergiant based on its optical spectrum. It seems paradoxical, but high-luminosity stars (hereafter - HLSs), which are primarily distinguished by their masses (the most massive stars with initial masses exceeding $20 \div 40 \mathcal{M}_{\odot}$, and intermediate-mass star with initial masses of $3 \div 9 \mathcal{M}_{\odot}$ ) and evolutionary stages, have similar observational properties: the properties of the optical and radio spectra, high IR excess, and complex and time-variable 
velocity field, which testify to instability of their extended atmospheres and expanding gas-dust envelopes. This intersection of observed properties for two types of objects leads to the problem of spectral mimicry. Stars in the groups indicated above, at fundamentally different stages of their evolution, display similar optical spectra and other properties (unstable and extended atmospheres, expanding gas-dust envelopes, high IR excesses). As a rule, according to the MK classification for post-AGB stars, these are high-luminosity objects (luminosity classes Ia, Ib, and II and absolute magnitudes $\mathrm{M}_{\mathrm{V}}$ from $-5^{\mathrm{m}}$ to $-7^{m}$ ). However, this actually only indicates a low value of $\log g$ in the photosphere (or pseudophotosphere) of the star.

For many years, we have studied stars with various masses with high mass-loss rates in their earlier and current stages of evolution. The fundamental reasons for mass loss and its evolutionary variations are not fully understood, underscoring the importance of determining the main characteristics of evolved stars and studying the structures of their extended envelopes and the parameters of their stellar winds. Our program includes studies of evolved stars with various masses: luminous blue variables (LBVs), which are very massive evolved stars near the Eddington stability limit; $\mathrm{B}[\mathrm{e}]$ stars, which are likely intermediate-mass binary systems soon after a stage of rapid mass transfer; white and yellow hypergiants; and low-mass post-asymptotic giant branch (post-AGB) supergiants with high IR excesses. The observed brightnesses of stars with circumstellar gas-dust envelopes in the visible is determined to an appreciable extent by the power of the envelope, which is related to the mass-loss rate. A necessary aspect of our studies, which is also the most labor intensive, is determining the luminosities and masses of the stars, in order to fix their evolutionary stage. When studying individual stars, we have observed similar, and even virtually identical, spectral features in different objects of the types indicated above.

In Section 2, we consider some aspects of the problem of spectral mimicry in more detail, using the properties of the objects listed in the table as examples. In Section 3, we suggest a means of resolving this problem. The vast majority of our studies were conducted using spectral material obtained using the same NES spectrograph $[1,2]$ of the $6-\mathrm{m}$ telescope of the Special Astrophysical Observatory (SAO) applying a single method for the spectral reduction. The uniformity of this observing material has enabled us to correctly compare features in the spectra of these different types of stars.

\section{Examples of spectral mimicry}

Let us briefly consider the main observational properties of two groups of HLSs: hypergiants and post-AGB stars which illustrate the problem of spectral mimicry. Hypergiants are extremely luminous objects. They are the descendents of high-mass stars with $\mathcal{M} \geq 20 \mathcal{M}_{\odot}$, and are observed during a short-lived stage in their evolution, making then very rare objects. According to the generally accepted view (see, e.g., the evolutionary scenarios of Conti [3]), these stars are observed as various types of HLSs during their evolution from the main sequence: white and yellow hypergiants, LBVs, B[e] stars, Wolf-Rayet stars. These very high-luminosity stars evolve, and lose matter at high rates, reaching $10^{-3} \mathcal{M}_{\odot} /$ yr in individual episodes. For example, according to [4], the mass-loss 
Table 1. Main data about the various types of supergiants considered. Spectral classes for a number of stars were taken from the SIMBAD database

\begin{tabular}{lllll}
\hline Star & IR source & Sp & Teff, log $g$ & Note \\
\hline Hypergiants & & & & \\
6 Cas & IRAS 23463+6156 & A2.5 Ia-0 & & {$[8]$} \\
HD 33579 & IRAS 05057-6756 & A3 IaO & $8129 \mathrm{~K} \mathrm{0.7}$ & {$[9]$} \\
V1302 Aql & IRC+10420 & A2Ia $^{+}$ & $8500 \mathrm{~K}, 1.0$ & {$[10]$} \\
HD 190603 & IRAS 20026+3204 & B5 Ia $^{+}$ & $18100 \mathrm{~K}, 2.41$ & {$[11]$} \\
Cyg OB2 No.12 & IRAS 20308+4104 & B5 Ia $^{+}$ & & {$[12]$} \\
Supergiants & & & & \\
HD 20902 $(\alpha$ Per $)$ & IRAS 03207+4941 & F5 Ib & $6579 \mathrm{~K} \mathrm{1.56}$ & {$[13]$} \\
HD 74180 & & Fo Ia & $7839 \mathrm{~K}, 2.11$ & {$[13]$} \\
HD 102878 & & A2 Iab & & SIMBAD \\
HD 148379 & & B2 Iab & & SIMBAD \\
HD 168571 & & BI Ib & & SIMBAD \\
post-AGB supergiants & & & \\
GSC 04501-00166 & IRAS 01005+7910 & B1.5 Ib & $21500 \mathrm{~K}, 3.0$ & {$[14,15]$} \\
BD +48 1220 & IRAS 05040+4820 & & $7900 \mathrm{~K}, 0.0$ & {$[16]$} \\
V510 Pup & IRAS 08005-2356 & F5 Ib-Iab & & {$[17]$} \\
V2324 Cyg & IRAS 20572+4919 & F0 III & $7500 \mathrm{~K}, 2.0$ & {$[18]$} \\
\hline
\end{tabular}

rate of HD 33579 is $10^{-5.7} \mathcal{M}_{\odot} / \mathrm{yr}$, and the mass-loss rate of $\mathrm{V} 1302 \mathrm{Aql}$ is $10^{-4.85} \mathcal{M}_{\odot} / \mathrm{yr}$. The star HD 190603, with a similar luminosity, loses mass at the rate $10^{-5.7} \mathcal{M}_{\odot} /$ yr $[5]$.

A determining factor in the evolution of these massive stars is their stellar winds, which create circumstellar gas-dust envelopes, which often have high densities and complex morphologies. The complex morphology of the circumstellar structures of evolved massive stars follow from radio spectroscopy data obtained in the bands of various molecules and masers (see, e.g., $[6,7]$ ).

In the course of their evolution, these stars supply the interstellar medium with matter freshly processed by nuclear reactions; they also influence the evolution of their host galaxies via their ionizing radiation and mechanical energy. White hypergiants are late B and early A stars with luminosity class Ia-0 $\left(\mathrm{M}_{\mathrm{V}}<-8^{\mathrm{m}}\right)$.

Only a few such objects are known in our Galaxy, including members of stellar associations: Cyg OB2 No. 12 (B5 Ia-0) [12], HD 168625 (B6 Ia-0), and HD 168607 (B9 Ia-0) in the Ser OB1A association [19]. Star No. 12 in the Cyg OB2 association is the best example of a hot hypergiant; its membership in this association implies the reliable luminosity $\log \left(L / L_{\odot}\right)=6.28$ [11]. The high luminosity of the star is also indicated by the high intensity of the OI $7773 \AA$ triplet. Its equivalent width is $\mathrm{W}_{\lambda}=1.14 \AA$ [12], which corresponds to the absolute magnitude $\mathrm{M}_{\mathrm{V}}<-8^{\mathrm{m}}$. This is one of the brightest stars in the Galaxy, and has the very high initial mass $\mathcal{M}=110 \mathcal{M}_{\odot}[11]$.

The properties of the stellar wind of Cyg OB2 No. 12 are clearly manifest in Fig. 1, which presents the $\mathrm{H} \alpha$ emission profiles in the spectra of this hypergiant obtained on the 6 -m telescope at various epochs in the interval $2001 \div 2011[12,20]$. The vertical dashed line indicates the velocity of the center of mass of the system (which we will refer to as 


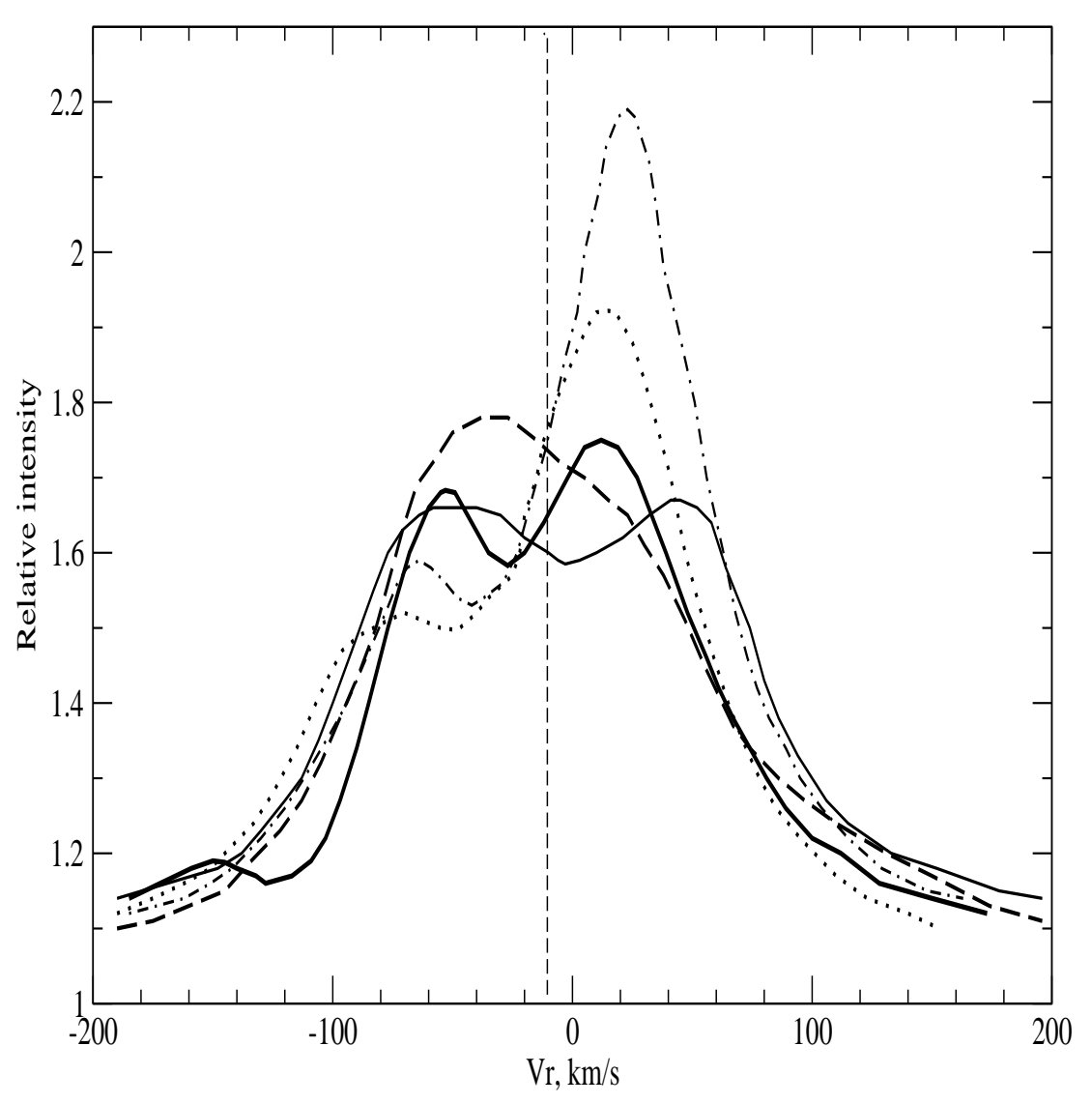

Figure 1. Variability in the $\mathrm{H} \alpha$ profile in spectra of the hot hypergiant star No. 12 in the Cyg OB2 association obtained on the 6-m telescope during 2001-2011 [12, 20]. The position of the vertical dashed line corresponds to the systemic velocity, Vsys $=-10.5 \mathrm{~km} / \mathrm{s}[20]$.

the systemic velocity), Vsys $=-10.5 \mathrm{~km} / \mathrm{s}[20]$. The diversity of these profiles reflects the instability of the hypergiant wind. Figure 1 shows that the profile shape is variable, but its main properties are preserved: powerful emission with a dip on the short-wavelength side, a jagged peak, and extended Thomson wings. The limiting wind speed is about $150 \mathrm{~km} / \mathrm{s}$. The inversion of the intensity in the upper part of the $\mathrm{H} \alpha$ profile testifies that the wind of Cyg OB2 No. 12 is inhomogeneous. In addition to the fast component noted above, the wind contains a fairly large amount of material that is nearly stationary relative to the star, or even falling onto the star. The coexistence in a single spectrum of lines with P Cygni and inverse P Cygni profiles, and even a combination of the two in a single line profile, has been noted for some LBVs at the phase of their maximum brightness [21]. This coexistence leads us to reject spherically symmetrical models for the wind.

Let us compare the $\mathrm{H} \alpha$ profile in the spectrum of star No. 12 in Cyg OB2 with the corresponding profiles in the spectra of supergiants of various masses. Figure 2 presents $\mathrm{H} \alpha$ profiles in the spectra of the stars we have chosen for comparison: the classical supergiants $\alpha$ Per and HD 102878, the A hypergiants HD 33579 and 6 Cas, and the post-AGB supergiants V510 Pup and BD $+48^{\circ} 1220$. For convenience in comparing the profiles for the 
various stars, the horizontal axis plots the shift $\Delta \mathrm{Vr}$ relative to the systemic velocity for each object. As expected, the $\mathrm{H} \alpha$ profiles in the spectra of the A hypergiants HD 33579 and 6 Cas are similar to the $\mathrm{H} \alpha$ profile of star No. 12. However, the emission-absorption profiles of this line in the spectra of the post-AGB supergiants V510 Pup and BD $+48^{\circ} 1220$ contain equally powerful emission. They are similar to the observed profiles in the spectra of hypergiants, but the velocity of the matter outflows are much lower in post-AGB stars. At the same time, the profiles of Population I supergiants (dashed curves in Figs. 2 and 3) differ sharply from both of these.

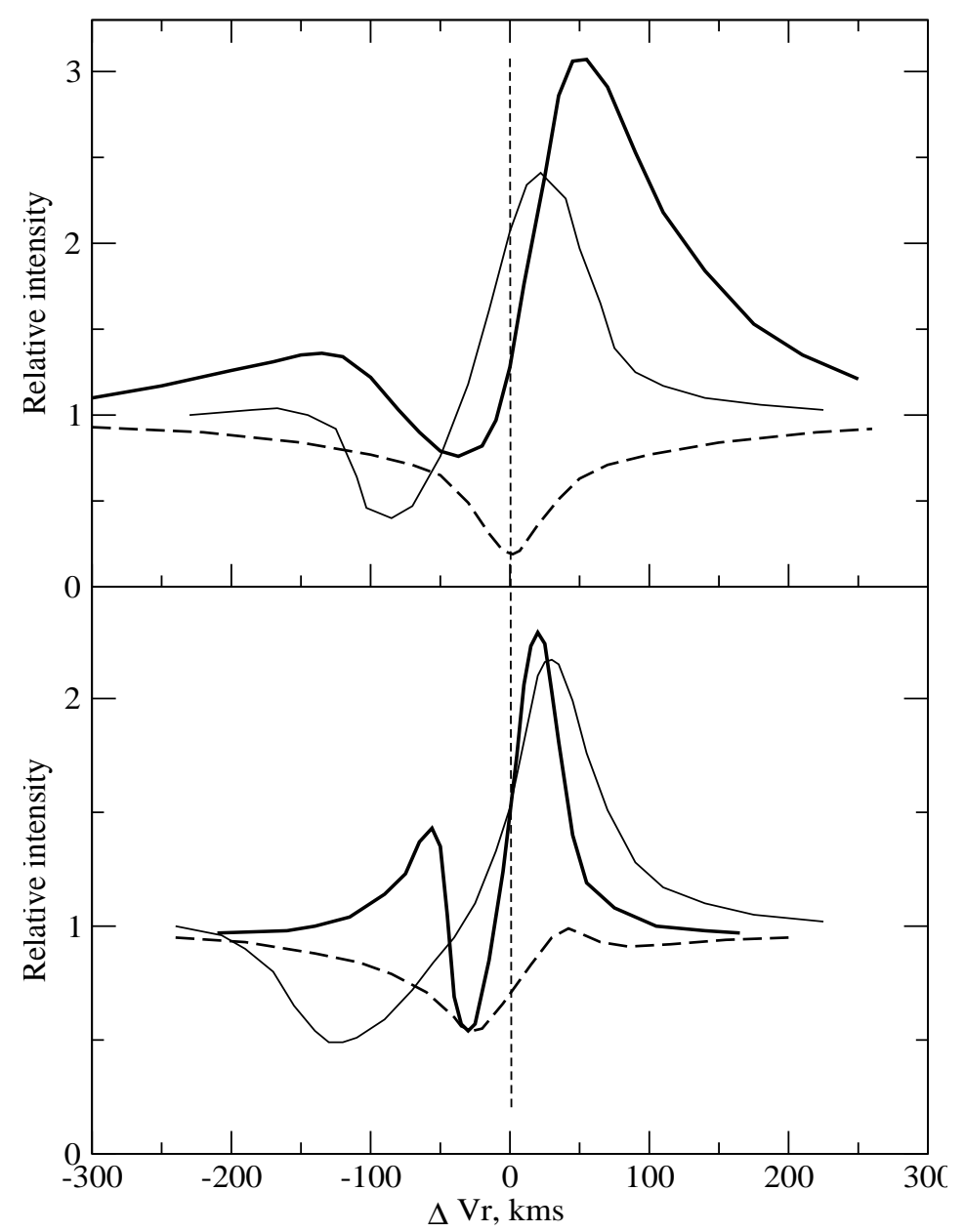

Figure 2. $\mathrm{H} \alpha$ profiles in the spectra of supergiants. The upper panel shows profiles for $\alpha$ Per (dashed), HD 33579 (thin), and V510 Pup (bold). The lower panel shows profiles for HD 102878 (dashed), 6 Cas (thin), and BD+48 1220 (bold). The vertical dashed line represents the systemic velocity of each object, and the horitonzal axis plots the shift $\Delta \mathrm{Vr}$ relative to the systemic velocity.

Given the well studied nature of the hypergiant 6 Cas and the fact that its spectral type is later than those of the supergiants listed above, we used this star in our comparisons with the spectra of A stars with other masses. Figure 2 shows that the longwavelength $\mathrm{H} \alpha$ absorption in 6 Cas makes a transition to strong emission, forming a P Cygni type profile, which is a sign of a spherically symmetrical wind. The mean velocity indicated by 


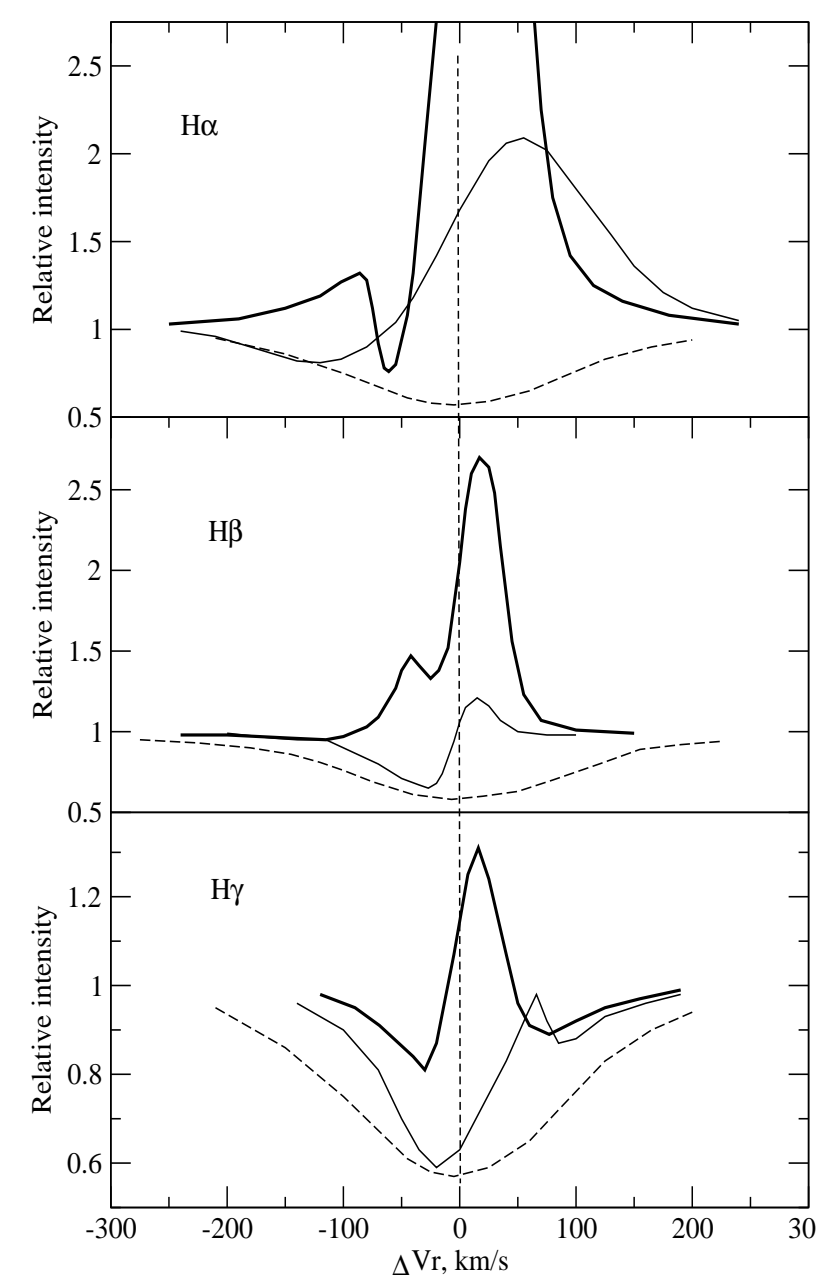

Figure 3. Neutral-hydrogen line profiles in the spectra of supergiants. The upper, middle and lower panels show profiles of $\mathrm{H} \alpha, \mathrm{H} \beta$, and $\mathrm{H} \gamma$, respectively. Results are shown for HD 168571 (dashed), HD 190603 (thin), and GSC 04501-00166 (bold). The horizontal axis plots the shift $\Delta \mathrm{Vr}$ relative to the systemic velocity of each object.

the broad absorption component is close to $\mathrm{Vr}=-150 \mathrm{~km} / \mathrm{s}$. The picture of a stable and spherically symmetrical wind is acceptable for white hypergiants in a first approximation, although with various caveats. For example, the main background component of the wind giving rise to the broad absorption features in the $\mathrm{H} \alpha$ P Cygni type profile in the spectrum of 6 Cas, which are deformed by moving dips, and the nearly symmetrical bell-like $\mathrm{H} \alpha$ profile in Cyg OB2 No. 12 (Fig. 1). Additional information is provided by Fig. 3, which presents $\mathrm{H} \alpha, \mathrm{H} \beta$, and $\mathrm{H} \gamma$ profiles in the spectra of stars with various luminosities.

While they appear to be analogs of post-AGB stars according to a number of their observational properties (high luminosity, peculiar spectral features, the presence of circumstellar molecular envelopes, etc.), yellow hypergiants have a fundamentally different nature. Their progenitors are massive (with initial masses $\geq 20 \mathcal{M}_{\odot}$ ) and have very high absolute magnitudes; after leaving the main sequence, they lose a substantial fraction of their mass and fall into the region of red supergiants, then into the region of yellow hypergiants. The typical luminosity of a yellow hypergiant is $\log \left(\mathrm{L}_{\mathrm{L}} \mathrm{L}_{\odot}\right) \approx 5.3 \div 5.9$ [22]. On 
the Hertzsprung-Russell diagram, these objects are located near the highest luminosities, in the region of instability containing hypergiants with spectral types from A to $\mathrm{M}[4,23]$. The structured circumstellar envelopes of hypergiants are formed through several episodes of strengthening of the powerful stellar wind (with mass-loss rates of $10^{-4} \div 10^{-3} \mathcal{M}_{\odot} / \mathrm{yr}$ ), and, as a rule, are powerful sources of IR and masers emission, as well as emission in various molecular lines.

Studies of hypergiants and searches for hypergiants in other galaxies are of special interest because it is precisely such stars that are believed to be the progenitors of massive SNe II and SNeIbc supernovae. The main steps in fixing the status of a hypergiant are determining its luminosity and mass loss parameters, identifying characteristic features in its optical spectral energy distribution, deriving the properties of its atmospheric abundances, and tracing variations in its parameters. This approach was applied to the known peculiar object IRC+10420, which is identified in the optical with the star V1302 Aql. Only after the discovery of a high nitrogen excess in its atmosphere in the late 1990s [10], together with the detection of dramatic time variations of its parameters, was this object finally classified as a yellow hypergiant [10, 24, 25, 27, 28]. Moreover, V1302 Aql has in recent years come to be considered the most convincing example of a yellow hypergiant, and its collected observational properties serve as a sort of template in studies of stars considered to be candidate yellow hypergiants (see, e.g., [29, 30]).

Distinguishing characteristics of the optical spectra of hypergiants are powerful emission (often twopeaked) in the Balmer and Paschen lines of hydrogen, which have broad wings due to Thomson scattering, and the presence of forbidden emission lines of metals. However, these two features are also present in the spectra of high-luminosity B[e] stars. The properties of these massive, hot supergiants can be found in [31,32], which present good examples of optical spectra obtained with high spectral resolution. The spectra of $\mathrm{B}[\mathrm{e}]$ stars are characterized by emission in lines of the CaII IR triplet and in the $\mathrm{Ca}[\mathrm{II}] 7291$ and $7324 \AA$ forbidden lines. These profiles are in good agreement with the hypothesis that a rotating circumstellar disk is present in a B[e] star system. The atlas [33], which compares the spectra of the B[e] star MWC 314 and the hypergiant V1302 Aql, is also useful for more detailed studies of $\mathrm{B}[\mathrm{e}]$-star spectra. We also recommend the study of Aret et al. [34], which contains a compilation of the observed properties of a sample of B[e] stars.

Thus, we must bear in mind that the combination of intense (often two-peaked) HI emission and forbidden emission lines of metals on its own does not provide a firm basis for classifying a star as a hypergiant. Spectral evidence that a star is a hypergiant is provided by the combination of the absorption spectrum of a supergiant with powerful HI emission and emission in forbidden lines of metals. A high luminosity, high massloss rate with a modest outflow speed, and a detailed analysis of the pattern of the radial velocities measured from lines with different origins are crucial when classifying a star as a hypergiant. This problem is illustrated clearly in the spectral atlas [33], which compares spectra of the B[e] star MWC 314 ( $\mathrm{Sp}=\mathrm{B} 3 \mathrm{Ibe}$ ) and the hypergiant V1302 Aql $\left(\mathrm{Sp}=\mathrm{F} 5 \mathrm{Ia}^{+} \mathrm{e}\right.$ at the epoch used to compile the atlas). These stars have different masses, luminosities, and spectral types, but have similar spectra. In particular, the powerful hydrogen emission lines have two-peaked profiles. The spectra both contain numerous permitted and forbidden emission lines of metals and features with P Cygni profiles. 
Stars on the asymptotic giant branch (AGB) have low-mass cores with typical masses $\approx 0.6 \mathcal{M}_{\odot}$, surrounded by an extended and often structured gas-dust envelope, which is formed via the loss of a substantial fraction of the stars mass in previous stages of its evolution. AGB stars make the transition to the planetary nebula phase with essentially constant luminosity, while becoming increasingly hotter. They have the observational characteristics of nebulae, and are often named as "protoplanetary nebulae" (PPN). One reason for the interest of both observers and theoreticians in AGB and post-AGB stars is that nucleosynthesis and the subsequent transport of carbon and heavy elements synthesized in the s-process to the stellar surface (third mixing) occur in these evolutionary stages [35]. As a result, intermediate-mass stars are the main suppliers to the interstellar medium of heavy elements (Sr, Y, Ba, La, and others), and also of a substantial fraction of $\mathrm{C}$ and $\mathrm{N}$ [36], which affect the chemical evolution of the galaxy.

In spite of the differences in their masses, internal structures, and ages, intermediatemass stars (with initial masses of $3 \div 8 \mathcal{M}_{\odot}$ ) in advanced stages of their evolution have spectra similar to those of true (i.e., massive) hypergiants. Moreover, we can also see an analogy in the structures of hypergiants and post-AGB stars: in both cases, the object has an evolved core surrounded by a gas-dust envelope formed in prior stages of its evolution. Post-AGB stars cannot immediately be distinguished from supergiants or hypergiants based only on optical spectroscopy. These are most often $\mathrm{F}-\mathrm{G}$ stars, although objects with earlier spectral types are also encountered. The high luminosities of these objects correspond to MK luminosity classes Ia,b, and II. However, this only indicates a low surface gravity in the stellar photosphere (or pseudo-photosphere). Post-AGB stars are often located at high Galactic latitudes, which is not characteristic of massive stars. The main types of optical spectral features displayed by PPN are:

1. metallic absorption lines with low or moderate intensities, symmetrical profiles without visible distortions;

2. time variable neutral-hydrogen absorption and emission components;

3. strong metallic absorption lines with low excitation potentials for their lower levels, with their variable profiles often distorted by features arising in the envelope;

4. molecular absorption or emission bands, primarily for molecules containing carbon;

5. envelope components of the NaI, KI resonance lines;

6. narrow permitted or forbidden emission lines of metals formed in the envelope.

The presence of features $2 \div 6$ represents the main differences between the spectra of PPN and of classical massive supergiants [37]. It is clear that all this variety of spectral features is lost in spectra with low resolution.

The H $\alpha$ lines in PPN spectra display complex (a combination of emission and absorption components), time-variable profiles of various types: with an asymmetric core, normal or inverse P Cygni type profile, with two emission components in the wings. Combinations of similar features are also observed fairly often. The presence of $\mathrm{H} \alpha$ emission indicates a high mass-loss rate and is one criterion in searches for PPN. The mass-loss rates of 
AGB stars are two to three orders of magnitude lower than those of hypergiants. The maximum mass-loss rate, rarely achieved in the AGB stage, is $10^{-5} \mathcal{M}_{\odot} /$ yr (see, e.g., the data of [38]). In addition, the outflow velocities do not exceed about $10 \div 20 \mathrm{~km} / \mathrm{s}$ - an order of magnitude lower than the outflow velocities of hypergiants. Only in the so-called "superwind" phase (with a duration of several hundred years) in the transition from a spherically symmetrical wind in the AGB stage to a collimated outflow in the post-AGB stage does the mass-loss rate grow by an order of magnitude (see [39] and references therein).

The complex character of the variability of profiles of features in PPN spectra is due to the fact that binary and pulsational instability can be superposed on variability due to inhomogeneity of the wind. A compilation of data on the periods and types of pulsation is presented in [40]. The main properties of the optical spectra of PPN are clearly illustrated by the results of studies of the spectral variability of HD 56126 [41], and by the atlas [42] of its spectra. According to its collected observational properties (a typical twopeaked spectral energy distribution, the spectrum of an $\mathrm{F}$ supergiant with variable $\mathrm{H} \alpha$ absorption and emission profiles, the presence of Swan bands of the $\mathrm{C}_{2}$ molecule in the optical, which form in the outflowing extended envelope, high excesses of carbon and heavy metals synthesized in the s-processes and dredge-upped to the surface by mixing), this star can be considered as a canonical post-AGB star. The degree of variability of its $\mathrm{H} \alpha$ profile is surprising: as follows from Fig. 2 in the atlas [42], all the profile types listed above were encountered in observations with the SAO 6-m telescope obtained over about a decade: an asymmetric core, normal or inverse P Cygni type profile, two emission features in the wings.

The weak central stars of the PPN we are considering here have substantially different spectral types: GSC04501-00166 (=IRAS01005+7910) has spectral type $\mathrm{B} 1.5 \mathrm{Ib}$ [14], BD+48 1220 (=IRAS 05040+4820) has type A4 Ib [16], and V510 Pup (IRAS 08005-2356) has type F5 Ib-Iab with emission lines [17]. The $\mathrm{H} \alpha$ profiles of all three stars have similar shapes. Their emission-absorption profiles are P Cygni in type, with the emission component appreciably exceeding the continuum level. An important property of the $\mathrm{H} \alpha$ profiles in the spectra of these PPN is that the relative intensity of the $\mathrm{H} \alpha$ emission is close to, or even exceeds, the corresponding relative intensities in the spectra of hypergiants with similar spectral types. Of the post-AGB stars presented in the Table, IRAS $01005+7910$ provides the most striking example of spectral mimicry of a massive hypergiant by a low-mass star in the post-AGB stage. However, as follows from Figs. 2 and 3, the velocities of wind absorption features in post-AGB supergiants are a factor of two to three lower than those in massive stars. Such relatively low wind speeds are characteristic of hypergiants, rather than classical supergiants.

The profiles of metal lines in the spectra of PPN also differ sharply from the norm; this is clearly visible in Fig. 4, which compares profiles of the HeI $5876 \AA$ and SiII $6347 \AA$ lines in the spectra of the hypergiant HD 190603, the classical supergiant HD 168571, and the post-AGB star GSC 04501-00166. The relative intensities of the emission components of both lines in the spectrum of the post-AGB star appreciably exceed their intensity in the spectra of the both more massive supergiants. Similar behavior can be seen in Fig. 5, which presents fragments of the spectra of the massive F supergiant HD 74180 and the post-AGB star V510 Pup (IRAS 08005-2356), which have similar spectral types. 

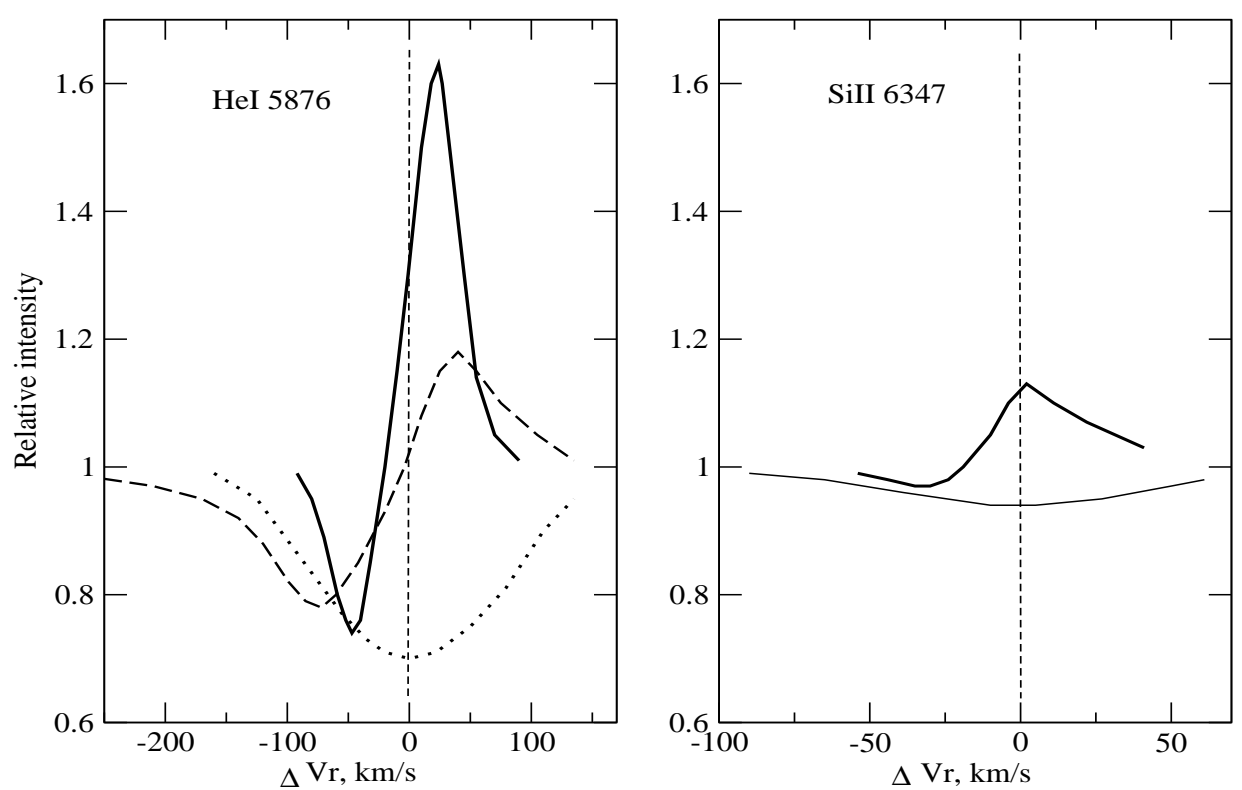

Figure 4. Profiles of selected lines in the spectra of supergiants: HeI 5876 å is shown to the left (the dotted profile is HD 168571, dashed is HD 190603, and bold is GSC 04501-00166), and SiII $6347 \mathrm{a}$ is shown to the right (the thin profile is HD 148379 and bold is GSC 04501-00166). The horizontal axis plots the shift $\Delta \mathrm{Vr}$ relative to the systemic velocity of each object.

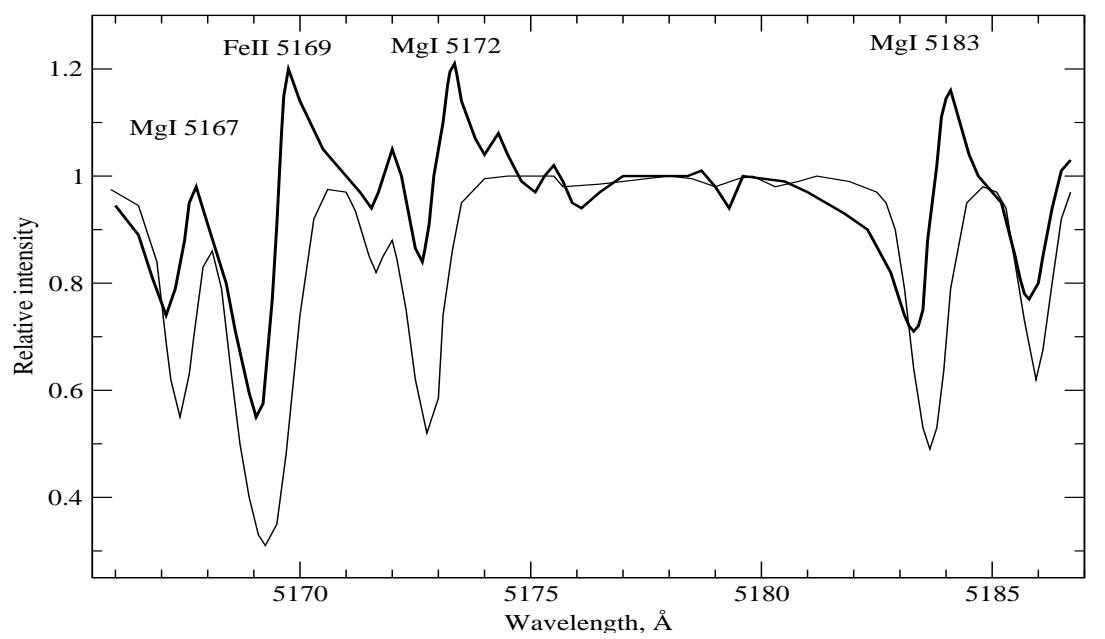

Figure 5. Comparison of fragments of the spectra of V510 Pup (bold) and HD 74180 (F0 Ia) (thin) at wavelengths $\lambda \lambda 5165 \div 5187 \AA$. The horizontal axis plots the laboratory wavelength.

In relation to the problem of mimicry, it is also useful to consider the observational properties of the poorly studied variable V2324 Cyg (it has visible magnitude $\mathrm{V}=11.63^{m}$, color indices $\mathrm{B}-\mathrm{V}=+1.09^{m}$ and $\mathrm{U}-\mathrm{B}=+0.58^{m}$, and Galactic coordinates $\mathrm{l}=89.44^{\circ}, \mathrm{b}=+2.39^{\circ}$, and the optical object is identified with the IR source IRAS 20572+4919). The observed excess at $12 \div 60 \mu$ and its position on an IR color diagram suggests that this object may be 
a young planetary nebula with a dust envelope [43]. Little is currently known about the properties of V2324 Cyg. Arkhipova et al. [44, 45] detected variability in the brightness of V2324 Cyg in longtime UBV observations, with amplitudes $\approx 0.3^{m}$ in $\mathrm{U}$ and $\approx 0.2^{\mathrm{m}}$ in $\mathrm{V}$ and $\mathrm{B}$. They explained the brightness variability as being due to the influence of the stellar wind. No pulsational periodicity was detected in the brightness variability [45]. The absence of pulsations is consistent with the fairly early spectral type of the star, A3 I [45]. Garcia-Lario et al. [46] used IR photometric data to determine the evolutionary statuses of 225 IRAS sources; they consider the classification of IRAS 20572+4919 as a post-AGB star to be tentative. A detailed study of the optical spectrum of V2324 Cyg was carried out in [18], where it is shown that V2324 Cyg has an atypically high rotational velocity for a post-AGB star, $V \sin i=69 \mathrm{~km} / \mathrm{s}$. The spectral type F2 III was determined for V2324 Cyg based on metallic lines of moderate intensity. The lower limit of the luminosity [18] leads to a contradiction with the properties of the chemical composition of its atmosphere. According to current thinking (see, e.g., the review [47]), large excesses of lithium and sodium provide evidence that the star is a post-AGB object which had an initial mass exceeding $4 \mathcal{M}_{\odot}$.

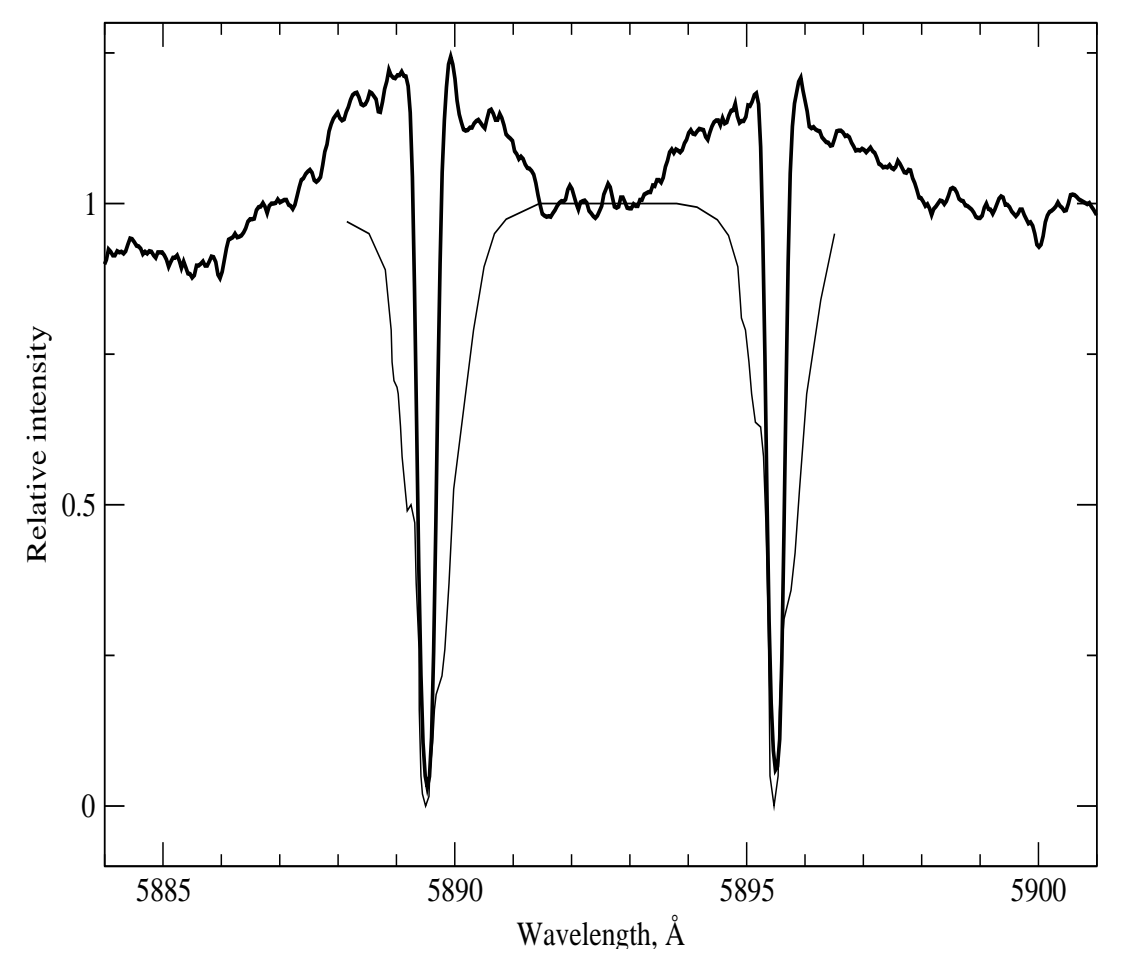

Figure 6. Comparison of profiles of the NaI D lines in the spectra of V2324 Cyg (F2 III) (bold) and HD 74180 (F0 Ia) (thin). The horizontal axis plots laboratory wavelength.

In connection with the publishing of the Gaia data, we are now able to refine the luminosity of V2324 Cyg. Taking into account interstellar reddening, $\mathrm{E}(\mathrm{B}-\mathrm{V})=1.1^{\mathrm{m}}$ [44], this object's parallax measured by Gaia, $\pi=1.55$ mas, implies a low absolute magnitude, $\mathrm{M}_{\mathrm{V}}=-1.0^{\mathrm{m}}$. However, some features of its spectrum sharply distinguish V2324 Cyg from ordinary early-subclass $\mathrm{F}$ giants, most importantly the presence of emission in a number of lines. As can be seen in Figs. 6 and 7, emission is present in the NaI (1) doublet, and is 


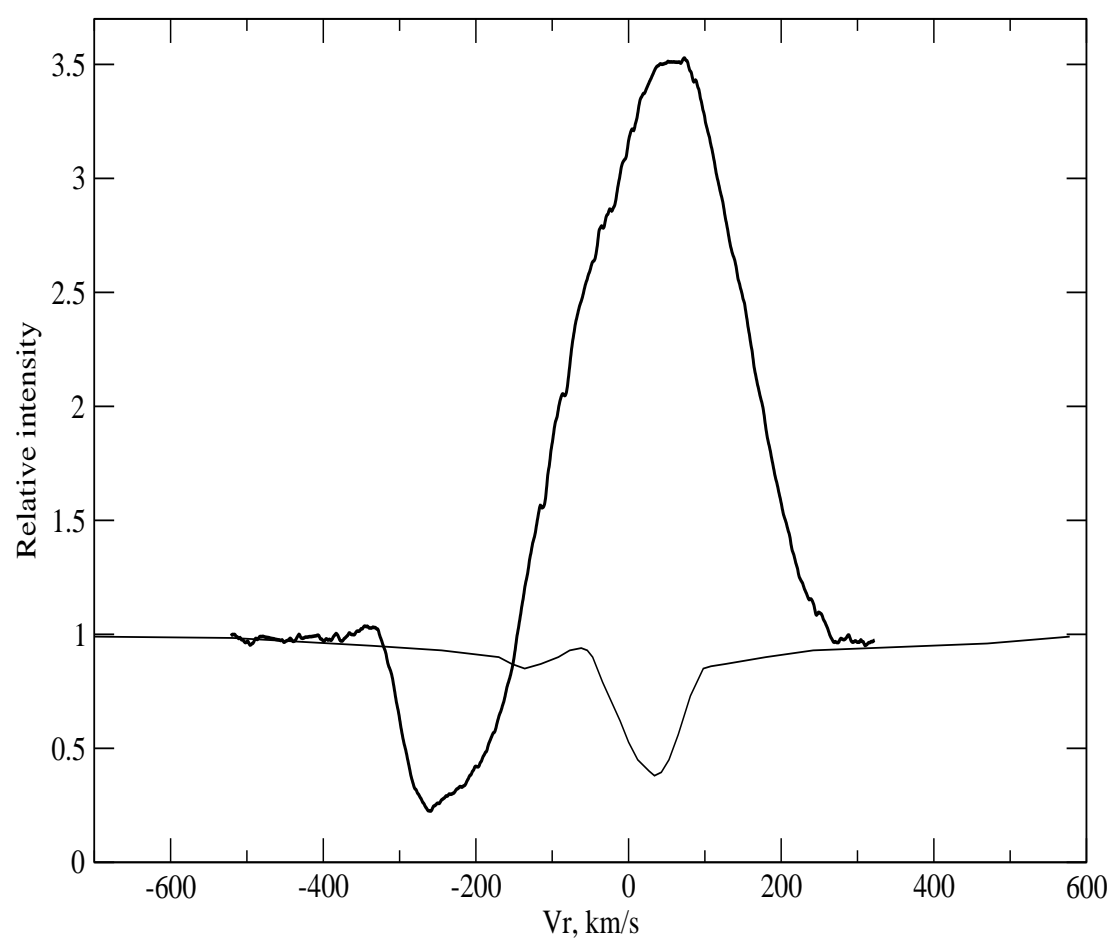

Figure 7. H $\alpha$ profile in the spectra of V2324 Cyg (F2 III) (bold) and HD 74180 (F0 Ia) (thin)

especially strong in $\mathrm{H} \alpha$ (the red component of its $\mathrm{P}$ Cygni type profile). The $\mathrm{H} \alpha$ emission peak exceeds the continuum level by a factor of three to four. A comparison of the $\mathrm{H} \alpha$ profiles in spectra obtained at different epochs indicates variability: the profile shape, emission intensity, and absorption depth all vary [18]. The positions of the emission peak and of absorption lines also vary. Overall, doubt still remains as to whether this star is in the post-AGB stage.

\section{Main results and conclusions}

The mass-loss rate grows with a star's luminosity, due to increasing density of the wind, since its speed is reduced. For example, according to [48], these parameters for $\alpha$ Cyg (A2 Ia), the very bright LMC white hypergiant HD 33579 (A3 Ia-0), and S Dor at their maximum brightnesses are related as follows: the mass-loss rate grows from the first to the last object as 1:100:1000, while the mean outflow velocities (based on rocketUV resonance lines) are 200, 120, and $70 \mathrm{~km} / \mathrm{s}$, respectively. This type of correlation is also obtained for our objects. Comparing the limiting outflow velocities derived from the $\mathrm{H} \alpha$ absorption lines and the mass-loss rates (from data in the literature), we conclude that the winds of supergiants and hypergiants are aspherical and nonstationary, with their spatial and kinematic structures simplifying with increasing luminosity [49]. Note that the question of spectral mimicry is one aspect of the general problem of fixing the evolutionary status of evolved stars with various masses. We note here the recent study of Humphreys et al. [50], who considered spectral mimicry among the most massive HLSs 
with optical emission lines: B[e] supergiants, LBVs, LBV candidates, and Wolf-Rayet stars.

A promising direction for studies of the final stages of the evolution of stars with various initial masses is joint studies and analyses of the chemical compositions and velocity fields in their atmospheres and envelopes. Ammonia $\left(\mathrm{NH}_{3}\right)$ was detected in the envelopes of the hypergiants V1302 Aql and HD 179821 [6], and the atmospheres of both these stars are enriched in nitrogen $[10,51]$. These results strongly suggest that both objects are descendents of massive stars. Similar results were also obtained for a number of post-AGB stars over the past decade. First, a subsample of post-AGB stars was distinguished, whose atmospheres display large excesses of carbon and heavy metals, and whose circumstellar envelopes have complex morphologies and are usually enriched in carbon, manifest through the presence of bands of $\mathrm{C}_{2}, \mathrm{C}_{3}, \mathrm{CN}, \mathrm{CO}$ and other carboncontaining molecules in the IR, radio, and optical spectra (see [37] and references therein). Second, several objects whose atmospheres are enriched in heavy metals are found among these post-AGB stars, for which high-resolution optical spectroscopy also indicates the presence of heavy metals in their circumstellar envelopes [37, 52-54]. We again emphasize that these spectral features cannot be studied in low-resolution, or even medium-resolution, spectra.

As a rule, fixing the evolutionary status of peculiar HLSs requires the use of a varied set of modern observational methods. This is clearly demonstrated by the history of studies of the peculiar supergiant V1302 Aql (IRC+10420), which was over several decades, right up to recent years, considered to be a post-AGB star. Only the collected results obtained over many years of observations using various methods on the largest optical and radio telescopes helped identify its true nature. In addition to high-resolution optical spectroscopy, decisive results were obtained from spectroscopy with high spatial resolution [24], IR spectroscopy [55], and spectropolarimetry [56]. Through these efforts, the object was finally classified as a massive star with extremely high luminosity - a yellow hypergiant. An example of an object with an uncertain status is the rather poorly studied variable A supergiant V2324 Cyg, identified with the IR source IRAS 20572+4919. The collected information that is available is currently insuffcient to unambiguously classify this as a massive or low-mass supergiant.

The reasons the problem of spectral mimicry are caused to the fact that essentially all features in the optical spectra of HLSs form in various layers of the extended and expanding stellar atmosphere. In recent studies analyzing spectra of very evolved stars of various spectral types with mass loss (see, e.g., [10, 57-60]), this extended atmosphere that is optically thick in the continuum and is formed by the stellar wind has sometimes been called a "pseudo-photosphere", following the introduction of this term in [61]. The presence of these specific layers is characteristic of both hypergiants and post-AGB stars. The total radiation flux determining the effective temperature forms in inner, energetically active layers of the star, but it is difficult to determine from spectral observations which energy-release processes give rise to the stellar radiation with this effective temperature: burning of hydrogen or helium in the core or in shell sources. Apart from their similar effective temperatures, these processes affect appreciably different luminosities. Another important factor influencing the formation of spectral features is the dynamical state of the atmosphere, in particular, the presence of an intense stellar wind and the passage of shocks resulting from pulsations. The variety of types of spectral features is determined 
primarily by the physical conditions in the extended atmosphere of the star, and depends less on the mass of lower-lying photospheric layers. This is clearly demonstrated by the presence of powerful HI emission lines in the spectra of post-AGB stars, whose relative intensities often exceed the corresponding relative intensities in the spectra of hypergiants.

In conclusion, we will focus on the known fact that HLSs are widely used as standard candles to construct a distance scale both in our Galaxy and extending to extragalactic distances. The spectral mimicry of supergiants must especially be borne in mind when using HLSs to determine the distances of distant objects. Unreliable estimates of an object's luminosity, and consequently its distance, can be obtained if one relies purely on the results of spectral classification. The luminosity of a star can be determined both from its membership in some group at a known distance, or from the dynamical properties of its atmosphere. The former method is rarely used, since stars located in short-lived evolutionary stages are very rarely encountered in clusters and associations. When applying the latter method, we often encounter the problem of spectral mimicry, when the same type of spectrum can be associated with objects with very different evolutionary statuses. The evolutionary status can be refined based on the changed chemical composition of the atmosphere (if nucleosynthesis products have already been transported to it). Few such cases are known (they comprise $67 \%$ of the sample studied in [54]). A second method is long-term spectroscopic monitoring, which can reveal properties of the pseudo-photosphere that cannot be determined from a single spectrogram.

As we have already noted above, the most important, but also the most difficult, aspect of our studies is fixing the luminosities of the stars. There is now hope that this problem will be eased even for distant objects with the accumulation and publication of astrometric data from the Gaia mission. Experience working with numerous and varied stellar spectra suggests another effective means of resolving this problem, which is unfortunately resourceintensive: detailed studies of the profiles of interstellar lines (NaI, DIBs, etc.) in the spectra of neighboring stars as a function of their distance in the Galaxy. This approach was successfully applied in studies of the anomalously red star No. 12 an LBV candidate in the Cyg OB2 association [62]. With the aim of clarifying the membership of stars in the association, classifying their spectra, determining their reddening, and investigating the behavior of reddening within the association, Maryeva et al. [62] obtained extensive additional spectral material for probable members of the association. A result of this study is the conclusion that the excess reddening of star No. 12 arises in its circumstellar envelope, which formed via high mass loss in prior evolutionary phases.

The resolution of the problem of mimicry for each individual object consists first and foremost in determining its evolutionary status, which requires determining and comparing various parameters: position in the Galaxy, luminosity, wind parameters, spectral energy distribution, chemical composition, and a detailed picture of the velocities at different levels in the stellar atmosphere and in its circumstellar medium.

Acknowledgements. Observations on the SAO 6-m telescope are supported by the Ministry of Education and Science of the Russian Federation (agreement No.14.619.21.0004, project code RFMEFI61914X0004). This work has made use of the SIMBAD and ADS astronomical databases and data from the ESA mission Gaia (https://www.cosmos.esa.int/gaia). 


\section{References}

1. V.E. Panchuk, V.G. Klochkova, M.V. Yushkin, and I.D. Naidenov, J. Opt. Technol. 7687 (2009).

2. V.E. Panchuk, V.G. Klochkova, and M.V. Yushkin, Astron. Rep. 61820 (2017).

3. P.S. Conti, Mem. Soc. Roy. Sci. Liège, 9193 (1975).

4. C. de Jager, Ann. Rev. Astron. Astrophys. 8145 (1998).

5. N. Markova and J. Puls, Astron. Astrophys.478 823 (2007).

6. G. Quintana-Lacaci, V. Bujarrabal, and A. Castro-Carrizo, Astron. Astrophys. 488203 (2008).

7. D. Teyssier, G. Quintana-Lacaci, A. P. Marston, V. Bujarrabal, et al., Astron. Astrophys. 545 A99 (2012).

8. E.L. Chentsov, Astrophys. Space Sci. 232217 (1995).

9. B. Wolf, Astron. Astrophys. 20275 (1972).

10. V.G. Klochkova, E.L. Chentsov, and V.E. Panchuk, MNRAS 29219 (1997).

11. J.S. Clark, F. Najarro, I. Negueruela, B.W. Ritchie, M.A. Urbaneja, and I.D. Howarth, Astron. Astrophys. 541 A145 (2012).

12. V.G. Klochkova and E.L. Chentsov, Astron. Rep. 481005 (2004).

13. R.E. Luck, Astron. J. 147137 (2014).

14. V.G. Klochkova, E.L. Chentsov, V.E. Panchuk, E.G. Sendzikas, and M.V. Yushkin, Astrophys. Bull. 69439 (2014).

15. V.G. Klochkova, M.V. Yushkin, A.S. Miroshnichenko, V.E. Panchuk, and K. Bjorkman, Astron. Astrophys. 392143 (2002).

16. V.G. Klochkova, E.L. Chentsov, N.S. Tavolzhanskaya, and V.E. Panchuk, Astron. Rep. 51 $642(2007)$.

17. V.G. Klochkova and E.L. Chentsov, Astron. Rep. 48301 (2004).

18. V.G. Klochkova, E.L. Chentsov, and V.E. Panchuk, Astrophys. Bull. 63112 (2008).

19. E.L. Chentsov and O.V. Maryeva, Astrophys. Bull. 71279 (2016).

20. E.L. Chentsov, V.G. Klochkova, V.E. Panchuk, M.V. Yushkin, and D.S. Nasonov, Astron. Rep. 57527 (2013).

21. O. Stahl, T. Gaeng, C. Sterken, A. Kaufer, T. Rivinius, T. Szeifert, and B. Wolf, Astron. Astrophys. 400279 (2003).

22. T.J. Jones, R.M. Humphreys, R.D. Gehrz, G.F. Lawrence, et al., Astrophys. J. 411323 (1993). 
23. H. Nieuwenhuijzen, C. de Jager, I. Kolka, G. Israelian, A. Lobel, E. Zsoldos, A. Maeder, and G. Meynet, Astron. Astrophys. 546 A105 (2012).

24. R. M. Humphreys, K. Davidson, and N. Smith, Astron. J. 1241026 (2002).

25. V. G. Klochkova, M. V. Yushkin, E. L. Chentsov, and V. E. Panchuk, Astron. Rep. 46139 (2002).

26. R.D. Oudmaijer, B. Davies, W.-J. de Wit, and M. Patel, in The Biggest, Baddest, Coolest Stars, Ed. by D.G. Luttermoser, B.J. Smith, and R.E. Stencel, ASP Conf Ser. 41217 (2009).

27. V.G. Klochkova, E.L. Chentsov, A.S. Miroshnichenko, V.E. Panchuk, and M.V. Yushkin, MNRAS 4594183 (2016).

28. R.M. Humphreys, N. Smith, K. Davidson, T.J. Jones, R.T. Gehrz, C.G. Mason, T.L. Hayward, J.R. Houck, and J. Krautter, Astron. J. 1142778 (1997).

29. R.M. Humphreys, K. Davidson, S. Grammer, N. Kneeland, J.C. Martin, K. Weis, and B. Burggraf, Astrophys. J. 77346 (2013).

30. J.S. Clark, I. Negueruela, B.W. Ritchie, and C. Gonzalez-Fernandez, Astron. Astrophys. 561 A15 (2014).

31. E.L. Chentsov, V.G. Klochkova, and A.S. Miroshnichenko, Astrophys. Bull. 65150 (2010).

32. V.G. Klochkova and E.L. Chentsov, Astrophys. Bull. 7133 (2016).

33. E.L. Chentsov, V.G. Klochkova, and N.S. Tavolganskaya, Bull. SAO 4825 (1999).

34. A. Aret, M. Kraus, and M. Slechta, MNRAS 4561424 (2016).

35. F. Herwig, Ann. Rev. Astron. Astrophys. 43435 (2005).

36. B. Gustafsson and N. Ryde, in IAU Symposium 177: The Carbon Stars Phenomenon, Ed. by R. Wing (Kluwer Academic, Dordrecht), p. 481 (2000)

37. V.G. Klochkova, Astrophys. Bull. 69279 (2014).

38. N. Mauron and P.J. Huggins, Astron. Astrophys. 452257 (2006).

39. B.L. de Vries, J.A.D.L. Blommaert, L.B.F.M. Waters, C. Waelkens, M. Min, R. Lombaert, and H. van Winckel, Astron. Astrophys. 561 A75 (2014).

40. L.L. Kiss, A. Derekas, Gy.M. Szabò, T.R. Bedding, and L. Szabados, MNRAS 3751338 (2007).

41. D. Barthes, A. Lebre, D. Gillet, and N. Mauron, Astron. Astrophys. 359168 (2000).

42. V.G. Klochkova, E.L. Chentsov, N.S. Tavolganskaya, and M.V. Shapovalov, Astrophys. Bull. $62162(2007)$.

43. P. Garcia-Lario, A. Manchado, S.R. Pottasch, J. Suso, and R. Olling, Astron. Astrophys. Supp. Ser. 82497 (1990).

44. V.P. Arkhipova and N.P. Ikonnikova, Astron. Lett. 20603 (1994). 
45. V.P. Arkhipova, N.P. Ikonnikova, R.I. Noskova, and G.V. Sokol, Astron. Lett. 26609 (2000).

46. P. Garcia-Lario, A. Manchado, W. Pych, and S.R. Pottash, Astron. Astrophys. Supp. Ser. 126479 (1997).

47. O. Straniero, R. Gallino, and S. Cristallo, Nucl. Phys. 777311 (2005).

48. O. Stahl, B. Wolf, O. Aab, and J. Smolinski, Astron. Astrophys. 252693 (1991).

49. E. L. Chentsov, Doctoral (Phys. Math.) Dissertation (Nizhnii Arkhyz, 2004).

50. R.M. Humphreys, K. Davidson, S. Grammer, N. Kneeland, J.C. Martin, K. Weis, and B. Burggraf, Astrophys. J. 83664 (2017).

51. T. Sahin, D. Lambert, V.G. Klochkova, and V.E. Panchuk, MNRAS 4614071 (2016).

52. V.G. Klochkova, Astron. Lett. 39765 (2013).

53. V.G. Klochkova, in Stars: From Collapse to Collapse, Ed. by Yu.Yu. Balega, D.O. Kudryavtsev, I.I. Romanyuk, and I.A. Yakunin, ASP Conf. Ser. 510121 (2017).

54. V.G. Klochkova and V.E. Panchuk, Astron. Rep. 60344 (2016).

55. R.D. Oudmaijer and W.-J. de Wit, Astron. Astrophys. 551 A69 (2013).

56. M. Patel, R.D. Oudmaijer, J.S. Vink, J.E. Bjorkman, B. Davies, M.A.T. Groenewegen, A.S. Miroshnichenko, and J.C. Mottram, MNRAS 385967 (2008).

57. P. Massey, PASP 112144 (2000).

58. B. Kaminsky and Ya.V. Pavlenko, MNRAS 35738 (2005).

59. T.N. Tarasova and A. Skopal, Astron. Rep. 56218 (2012).

60. R. Klement, A.C. Carcio, T. Rivinius, D. Panoglou, et al., Astron. Astrophys. 584 A85 (2015).

61. C. Leitherer, I. Appenzeller, G. Klare, H.J.G.L.M. Lamers, O. Stahl, L.B.F.M. Waters, and B. Wolf, Astron. Astrophys. 551168 (1985).

62. O.V. Maryeva, E.L. Chentsov, V.P. Goranskij, V.V. Dyachenko, S.V. Karpov, E.V. Malogolovets, and D.A. Rastegaev, MNRAS 458491 (2016). 